\title{
Sequential Bayesian Inference for Detection and Response to Seasonal Epidemics
}

\author{
Michael Ludkovski* and Junjing Lin \\ Statistics and Applied Probability, UC Santa Barbara, Santa Barbara, CA, USA
}

\section{Objective}

Development of a sequential Bayesian methodology for inference and detection of seasonal infectious disease epidemics.

\section{Introduction}

Detection and response to seasonal outbreaks of endemic diseases provides an excellent testbed for quantitative bio-surveillance. As a case study we focus on annual influenza outbreaks. To incorporate observed year-over-year variation in flu incidence cases and timing of outbreaks, we analyze a stochastic compartmental SIS model that includes seasonal forcing by a latent Markovian factor. Epidemic detection then consists in identifying the presence of the environmental factor ("high" flu season), as well as estimation of the epidemic parameters, such as contact and recovery rates.

\section{Methods}

Anticipating policy-making applications, we consider sequential Bayesian inference. To focus on intrinsic model uncertainty, we assume full observation of all individual status changes, but unobserved seasonal factor $\mathrm{M}$ underscore " $\mathrm{t}$ " and unknown reaction rates. Using theory of nonlinear filtering of point processes, we derive analytic expressions for conditional likelihoods of latent factor trajectories. We then utilize a Sequential Monte Carlo approach based on Particle Learning (PL) [1] to infer the epidemic parameters in conjunction with online filtering of M underscore "t." These tools extend the PL method to continuous-time jump-Markov models and are widely applicable in generic stochastic chemical kinetic models.

Using the developed inference methods, we then investigate costefficient sequential policy making. We analyze and compare several heuristic counter-measure strategies that work by modifying the duration/frequency of the high epidemic season.

\section{Results}

The proposed algorithm was implemented in R and extensively tested on simulated data [2]. We find that the PL method is able to efficiently carry out joint inference. We also find that counter-measures incorporating sequential learning are generally more efficient that other inference-free policies.

\section{Conclusions}

We developed a new Bayesian approach to joint inference of parameters and latent factors in continuous-time stochastic compartmental models. There is ongoing work [3] to adjust our methods for more realistic observation schemes.
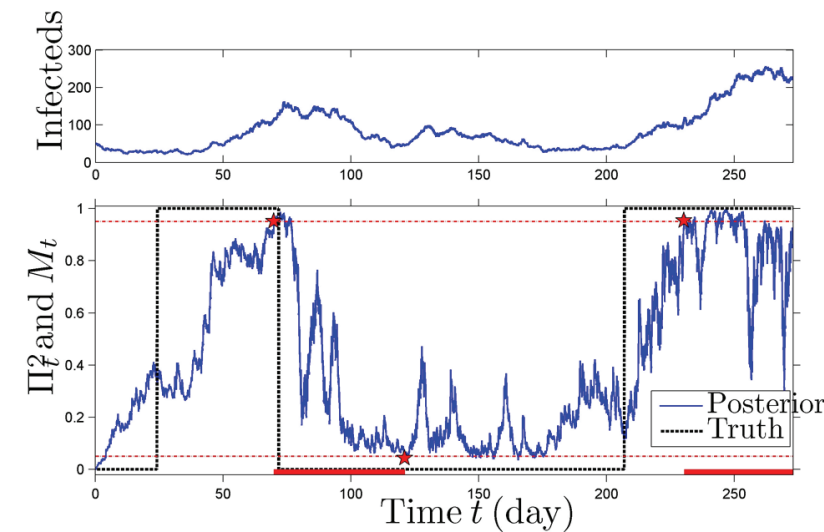

Fig: Response strategy based on the Bayesian posterior probability $\$ \backslash \mathrm{Pi}^{\wedge} 2 \_\mathrm{t} \$$ (use math notation) of high flu season $\$ \backslash\left\{\mathrm{M}_{-} \mathrm{t}=1 \backslash\right\} \$$. Countermeasures begin when prob $>95 \%$ and end when prob $<5 \%$.

\section{Keywords}

Bayesian inference; stochastic compartmental models; seasonal epidemics; hidden Markov models

\section{Acknowledgments}

We thank Jarad Niemi for useful discussions.

\section{References}

[1] Carvalho, C. M.; Johannes, M.; Lopes, H. F. and Polson, N. Particle learning for sequential Bayesian computation. Bayesian Statistics, 2011, 9, 317-360.

[2] Lin J, and Ludkovski M., Sequential Bayesian Inference in Hidden Markov Stochastic Kinetic Models with Application to Detection and Response to Seasonal Epidemics, Submitted, 2012.

[3] Ludkovski M, Niemi J. Optimal disease outbreak decisions using stochastic simulation. In: Proceedings of the 2011 Winter Simulation Conference. Jain S, et al eds.

*Michael Ludkovski

E-mail: ludkovski@pstat.ucsb.edu 